\title{
Características clínicas de mujeres con depresión mayor que sufrieron abuso sexual en la infancia
}

\author{
José A. Ortiz-Guzmán, ${ }^{1}$ María C. Ibarra-Alcantar, ${ }^{1,2}$ Francisco J. Alvarado-Cruz, ${ }^{1}$ \\ Hermelinda Graciano-Morales ${ }^{1}$ y Alejandro Jiménez-Genchi ${ }^{3}$ \\ ${ }^{1}$ Centro Comunitario de Salud Mental; ${ }^{2}$ Hospital Psiquiátrico Fray Bernardino Álvarez; ${ }^{3}$ Secretaría de Salud, Instituto Nacional de Psiquiatría Ramón \\ de la Fuente. Ciudad de México, México
}

\section{Resumen}

Introducción: Aunque el abuso sexual en la infancia (ASI) es un factor de riesgo para desarrollar depresión mayor en la vida adulta de las mujeres, la información sobre las características clínicas asociadas con este evento es escasa. Objetivo: Evaluar la historia de ASI y su asociación con algunas manifestaciones clínicas en mujeres con depresión mayor. Método: Se seleccionaron 71 mujeres adultas con depresión mayor en un centro comunitario de salud mental. Fueron evaluadas con Mini Entrevista Neuropsiquiátrica Internacional, Inventario de Depresión de Beck, Escala de Autoevaluación de Adaptación Social, Índice de Maltrato Físico e Índice de Abuso Sexual en la Infancia. Resultados: 53.5 \% había sufrido alguna forma de ASI. No hubo diferencias significativas en edad, escolaridad, sintomatología depresiva, adaptación social o maltrato físico en la infancia entre las mujeres con y sin historia de ASI, solo mayor frecuencia de comorbilidad con el trastorno por ansiedad social (26 versus $6 \%$ ) y mayor proporción de riesgo suicida (68 versus 45.4 \%) distinguió a los grupos. Conclusión: Pocas características distinguen a las mujeres con depresión mayor con historia de ASI, sin embargo, el pronóstico y las implicaciones terapéuticas del mayor riesgo suicida subrayan la importancia de investigar sistemáticamente la historia de ASI entre las mujeres con depresión mayor.

PALABRAS CLAVE: Depresión mayor. Abuso sexual infantil. Maltrato físico. Riesgo suicida. Funcionamiento social.

\begin{abstract}
Introduction: Even when child sexual abuse (CSA) is a risk factor for the development of major depression in adult women, data on the clinical features associated with this traumatic event are scarce. Objective: To assess the history of CSA and its association with some clinical manifestations in women with major depression. Method: Seventy-one adult women with major depression were selected in a community-based mental health center. They were assessed with the Mini International Neuropsychiatric Interview, the Beck Depression Inventory, the Social Adaptation Self-evaluation Scale, and childhood physical maltreatment and child sexual abuse indices. Results: Some form of CSA had been suffered by 53.5\%. There were no significant differences in age, level of education, depressive symptoms, social adaptation or physical maltreatment during childhood between the women with or without a history of CSA, only a higher frequency of comorbid social anxiety disorder (26\% versus $6 \%$ ) and a higher proportion of suicide risk (68\% versus $45.4 \%$ ) differentiated the groups. Conclusion: Few characteristics distinguish women with major depression with a history of CSA; however, the prognosis and therapeutic implications of a higher suicide risk underscore the importance of systematically investigating the history of CSA among women with major depression.
\end{abstract}

KEY WORDS: Major depression. Child sexual abuse. Physical maltreatment. Suicide risk. Social functioning.

Correspondencia: Alejandro Jiménez-Genchi

E-mail: jimalex@imp.edu.mx
Fecha de recepción: 25-01-2017

Fecha de aceptación: 06-02-2018

DOI://dx.doi.org/10.24875/GMM.18003221
Gac Med Mex. 2018;154:295-301

Disponible en PubMed

www.gacetamedicademexico.com 


\section{Introducción}

El abuso sexual en la infancia (ASI) es una de las experiencias adversas más traumáticas y con mayor impacto en el desarrollo emocional de un individuo. ${ }^{1-3}$

De acuerdo con una revisión de la epidemiología internacional de este fenómeno, a fines del siglo XX entre 7 y $36 \%$ de las mujeres había sufrido ASI; ${ }^{1}$ específicamente se estimaba que en Estados Unidos una de cada cinco mujeres había sido víctima de ASI. En México, la Encuesta Nacional sobre Violencia contra las Mujeres 2003 encontró una prevalencia de $7.3 \%$ en mujeres usuarias de servicios de atención primaria y del segundo nivel. ${ }^{2}$ Por su parte, la Encuesta de Maltrato Infantil y Factores Asociados 2006 llevada a cabo en adolescentes de Baja California, Sonora, Tlaxcala y Yucatán reportó una prevalencia de abuso sexual entre 3.5 y $9.1 \%$ en la población femenina. ${ }^{3}$

Un buen número de estudios y varias revisiones metaanalíticas han documentado de manera consistente que el ASI se asocia con el desarrollo de trastornos psiquiátricos en la vida adulta. ${ }^{4}$ Este efecto parece ser particularmente más pronunciado en las mujeres, predisponiéndolas a mayor número de trastornos psiquiátricos en comparación con los hombres. ${ }^{5}$ La asociación más estudiada ha sido la existente entre el ASI y el trastorno depresivo mayor, identificándose al primero como un sólido factor de riesgo para la aparición del segundo, incluso atribuyéndosele algún efecto causal. ${ }^{6,7}$

Pero no solamente es importante la contribución del ASI a la causalidad de la depresión, sino también puede serlo para la configuración de perfiles sintomáticos de los episodios depresivos, aunque la información es menor en este rubro. Se ha encontrado que las mujeres con depresión e historia de ASI desarrollan el primer episodio depresivo a una edad más temprana, presentan más conductas autolesivas, conducta suicida e impulsivas, ${ }^{8-10}$ más trastornos psiquiátricos comórbidos ${ }^{11}$ y se encuentran en mayor riesgo de sufrir maltrato físico y sexual en la edad adulta. ${ }^{10}$

Pocos estudios han evaluado la comorbilidad psiquiátrica, el funcionamiento social y la asociación con otros tipos de maltrato en las mujeres con depresión mayor e historia de ASI. Cuando se ha investigado la relación con características clínicas tales como la gravedad de los síntomas depresivos se han obtenido resultados poco consistentes. En virtud de lo anterior, el presente trabajo tuvo como objetivo evaluar la historia de abuso sexual en la infancia y su asociación con algunas manifestaciones clínicas, como la gravedad de los síntomas depresivos, el riesgo suici$\mathrm{da}$, el funcionamiento social y la historia de maltrato físico en mujeres con trastorno depresivo mayor.

\section{Método}

\section{Participantes}

Se seleccionó una muestra no probabilística por conveniencia de la población que solicitó atención psiquiátrica en el Centro Comunitario de Salud Mental Cuauhtémoc de la Ciudad de México, entre el 4 de marzo y el 31 de agosto de 2005. Se invitó a participar solamente a las mujeres entre 18 y 65 años, alfabetas, que en la evaluación clínica de admisión recibieron el diagnóstico de trastorno depresivo mayor (de acuerdo con los criterios del DSM-IV) y que no habían recibido tratamiento farmacológico o psicoterapéutico en los últimos tres meses. Tras una explicación sobre las características y propósito del estudio, se les solicitó su consentimiento informado por escrito.

\section{Procedimiento}

A todas las pacientes se les realizó la Mini Entrevista Neuropsiquiátrica Internacional $(\mathrm{MINI})^{12}$ con la finalidad de confirmar el diagnóstico principal y documentar la comorbilidad psiquiátrica. Asimismo, se empleó una cedula de información para registrar los datos sociodemográficos y otras características clínicas de cada participante.

Las entrevistas fueron realizadas por psiquiatras con experiencia clínica y adiestramiento en la aplicación de la MINI. El proyecto de investigación fue aprobado por el comité de ética institucional. Después de concluir la entrevista diagnóstica, las participantes llenaron una serie de instrumentos de evaluación.

\section{Instrumentos}

El Inventario de Depresión de Beck (IDB) ${ }^{13}$ es un instrumento autoaplicable ampliamente utilizado en la investigación relativa a la depresión. Consta de 21 reactivos sobre diferentes síntomas depresivos, con cuatro opciones de respuesta para cada reactivo y cuyos valores van de cero a tres. Al sumar las puntuaciones en los reactivos se obtiene una medida global de la gravedad de la depresión, de tal forma la mayor puntuación corresponde a mayor gravedad. 
El Índice de Abuso Sexual en la Infancia (IASI) se desarrolló como parte de la investigación; los detalles del proceso se han descrito anteriormente..$^{14}$ En resumen, tres de los investigadores elaboraron un banco de reactivos cuyo fin era investigar una amplia variedad de experiencias de maltrato sufridas en la infancia. Cuatro clínicos expertos en la atención de sobrevivientes de maltrato fungieron como jueces y clasificaron los reactivos en varias categorías de maltrato. Los reactivos que al menos tres de los jueces consideraron como abuso sexual se emplearon para construir el IASI.

El cuestionario, el cual se presenta en el anexo, consta de ocho reactivos que investigan la historia y características de distintas experiencias de abuso sexual en la infancia (edad del sujeto, número de ocasiones que ocurrió, parentesco con el perpetrador del maltrato y la edad de este). El valor para cada reactivo se obtuvo a partir del número de ocasiones que el sujeto registró haber sufrido el abuso en cuestión. Se evaluaron algunas de sus propiedades psicométricas en una muestra de 115 mujeres. Los coeficientes de correlación entre cada reactivo y la suma total de ellos fueron significativos $(0.73,0.63,0.59,0.76,0.77$, $0.84,0.47$ y 0.28 , respectivamente; $p<0.01$ ). El coeficiente de confiabilidad en dicha muestra fue de 0.80 , mientras que en las participantes de este estudio fue de 0.78. En esta investigación se empleó una medición categórica del abuso sexual, en el que su presencia se definió como una calificación mayor que cero.

El Índice de Maltrato Físico Infantil (IMFI) también se construyó como parte de la investigación y paralelamente al IASI. Tiene un formato autoaplicable constituido por cinco reactivos que investigan la frecuencia con que un sujeto recibió golpes de sus padres o tutor cuando tenía entre tres y 17 años de edad. Las opciones de respuesta son nunca, algunas veces, frecuentemente y casi siempre, cuyos valores son $0,1,2$ y 3 , respectivamente. La suma de las puntuaciones en cada reactivo proporciona una calificación global para el maltrato físico. En una muestra clínica de mujeres con depresión mayor o distimia, el IMFI mostró un coeficiente de confiabilidad de $0.88 .{ }^{14}$ En la población de este estudio la confiabilidad también fue satisfactoria (0.90).

El riesgo suicida se determinó mediante el módulo correspondiente de la MINI. ${ }^{12}$ Este apartado está constituido por seis preguntas sobre la presencia en el último mes de deseos de muerte, autolesiones, ideas suicidas, planes de suicidio e intentos suicidas, así como la presencia de estos últimos a lo largo de toda la vida. Las opciones de respuesta son sí y no, correspondiendo a las afirmaciones los valores de 1 , $2,6,10,10$ y 4 puntos, respectivamente; al sumar estos valores se obtiene una puntuación global, que clasifica al riesgo suicida en leve (1-5 puntos), moderado (6-9 puntos) y alto ( $\geq 10$ puntos). En este estudio se empleó una definición dicotómica, considerándose la existencia de riesgo suicida cuando la calificación global era de 1 o más.

La Escala de Autoevaluación de la Adaptación Social (EAAS) consta de 21 reactivos que evalúan la conducta y motivación social en el área laboral y actividades placenteras, las relaciones familiares y extrafamiliares, los intereses intelectuales, la satisfacción con los roles y la autopercepción del paciente de su capacidad para manejar y controlar su ambiente..$^{15}$ Cada respuesta tiene un valor entre cero y tres, correspondientes a una mínima o máxima adaptación social; el rango de puntuación total se encuentra entre cero y 60 , indicando mayor adaptación social conforme aumenta la calificación. Se ha reportado que posee una confiabilidad aceptable (alfa de Cronbach = $0.74)$, adecuada estabilidad temporal y es sensible al cambio. La versión en español también ha mostrado propiedades psicométricas satisfactorias. ${ }^{16}$

\section{Análisis}

Los resultados se describen con medias y desviaciones estándar y proporciones, según sea el caso. Se emplearon pruebas t para comparar las puntuaciones en la sintomatología depresiva, maltrato físico en la infancia y adaptación social entre las mujeres con historia positiva de ASI versus historia negativa; el riesgo suicida se comparó con chi cuadrado o prueba exacta de Fisher. Se estableció como significativo un valor de $p<0.05$.

\section{Resultados}

Se evaluaron 76 mujeres, de las cuales se excluyeron cinco debido a que presentaban historia de episodios maníacos o hipomaníacos. De las 71 participantes incluidas en el estudio, $53.5 \%$ reportó haber sufrido alguna forma de ASI. En la Tabla 1 se presentan las distintas formas de abuso sexual que experimentaron las pacientes, así como el parentesco con el perpetrador; $81.5 \%(n=31)$ de las mujeres con ASI sufrió más de una forma de abuso entre los tres y 17 años, con una media de $8.3 \pm 2.2$ años. 
Gaceta Médica de México. 2018;154

Tabla 1. Parentesco del perpetrador del abuso sexual con la sobreviviente

\begin{tabular}{|c|c|c|c|c|c|c|c|c|c|}
\hline \multirow[t]{2}{*}{ Conducta de abuso } & \multirow[t]{2}{*}{$\mathrm{n}$} & \multicolumn{8}{|c|}{ (\%) } \\
\hline & & Padre & Padrastro & Hermano & Tío & Primo & Amigo & Vecino & Desconocido \\
\hline Quitarse la ropa & 23 & 17.3 & - & - & 17.3 & 30.4 & 4.3 & 8.6 & 21.7 \\
\hline Tocamientos & 24 & 12.5 & 4.1 & - & 29.1 & 29.1 & - & 4.1 & 20.3 \\
\hline Tocar al agresor * & 17 & 11.7 & - & 5.8 & 23.5 & 17.6 & - & 5.8 & 29.4 \\
\hline Dar besos & 10 & 10 & 10 & 20 & 10 & 20 & - & - & 30 \\
\hline Recibir besos & 13 & 7.7 & 7.7 & - & 23 & 23 & - & 7.7 & 30.7 \\
\hline Frotamientos & 21 & 9.5 & 9.5 & 4.7 & 14.2 & 33.3 & - & - & 28.5 \\
\hline $\begin{array}{l}\text { Intento de relación } \\
\text { sexual * }\end{array}$ & 15 & 26.6 & - & - & 13.3 & 20 & - & - & 26.6 \\
\hline Penetración* & 11 & - & - & - & 27.2 & 9.1 & - & - & 54.5 \\
\hline
\end{tabular}

*Para algunos agresores no se identificó el parentesco por lo que los porcentajes son menores a 100.

Al comparar las características demográficas, tales como edad y escolaridad, no se encontraron diferencias significativas entre las mujeres con historia de ASI y las que no lo presentaron. Tampoco se observaron diferencias en la gravedad de la sintomatología depresiva, el grado de adaptación psicosocial y la gravedad del maltrato físico. Solamente el trastorno por ansiedad social y la presencia de riesgo suicida fueron significativamente más frecuentes en las mujeres con historia positiva de ASI (Tabla 2).

\section{Discusión}

En esta muestra de mujeres con depresión mayor que acudieron a un centro comunitario de salud mental para recibir atención encontramos que la mitad de ellas había sido víctima de algún tipo de abuso sexual durante su infancia, lo cual además se asoció con mayor comorbilidad con trastorno de ansiedad social y riesgo suicida.

La proporción de mujeres con ASI es notablemente mayor a lo que se ha encontrado en México en mujeres usuarias de centros de atención primaria ${ }^{17}$ y de segundo nivel (7 a $21 \%),{ }^{2}$ población general (3.5-9.1\%) ${ }^{3}$ e incluso es mayor a $23-30 \%$ que se ha reportado en otros estudios con esta población clínica. ${ }^{9-11}$ Entre las explicaciones para esta diferencia están la población estudiada, es decir, una muestra clínica de mujeres con uno de los trastornos psiquiátrico más frecuentemente asociados con la historia de abuso sexual. ${ }^{7} Y$ en conjunto con esta característica, la definición de abuso sexual empleada. Los estudios que lo han definido como una relación sexual (penetración) en contra de la voluntad han reportado las cifras más bajas ${ }^{2,17}(7$ a $11 \%)$, mientras que las proporciones aumentan (21-29.4\%) cuando
Tabla 2. Características demográficas y clínicas de la muestra

\begin{tabular}{|c|c|c|c|c|c|c|}
\hline & \multicolumn{2}{|c|}{$\begin{array}{c}\text { Sin abuso } \\
\text { sexual } \\
(n=33)\end{array}$} & \multicolumn{2}{|c|}{$\begin{array}{c}\text { Con abuso } \\
\text { sexual } \\
(n=38)\end{array}$} & \multirow[t]{2}{*}{$\begin{array}{c}t \\
(g \mid 69)\end{array}$} & \multirow[t]{2}{*}{$p$} \\
\hline & Media & DE & Media & $\mathrm{DE}$ & & \\
\hline Edad (años) & 37.8 & 11.8 & 36.0 & 9.8 & 0.67 & 0.50 \\
\hline Escolaridad (años) & 10.1 & 3.6 & 10.0 & 3.1 & 0.22 & 0.82 \\
\hline $\begin{array}{l}\text { Inventario depresión } \\
\text { de Beck }\end{array}$ & 29.8 & 8.3 & 31.3 & 9.9 & -0.68 & 0.49 \\
\hline Adaptación social & 31.0 & 8.9 & 30.7 & 9.2 & 0.16 & 0.87 \\
\hline \multirow[t]{2}{*}{ Maltrato físico } & 3.6 & 4.5 & 5.5 & 4.4 & -1.7 & 0.08 \\
\hline & $\mathbf{n}$ & $\%$ & $\mathbf{n}$ & $\%$ & $\begin{array}{c}x^{2} \\
(g \mid 1)\end{array}$ & 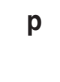 \\
\hline Riesgo suicida & 15 & 45.4 & 26 & 68 & 3.8 & 0.04 \\
\hline Trastorno de pánico & 8 & 24.2 & 13 & 34.2 & 0.84 & 0.25 \\
\hline Agorafobia & 4 & 12.1 & 6 & 15.7 & $\S$ & 0.46 \\
\hline $\begin{array}{l}\text { Trastorno de ansiedad } \\
\text { social }\end{array}$ & 2 & 6.0 & 9 & 23.6 & 4.1 & 0.04 \\
\hline $\begin{array}{l}\text { Trastorno obsesivo } \\
\text { compulsivo }\end{array}$ & 1 & 3.0 & 0 & 0 & $\S$ & 0.46 \\
\hline $\begin{array}{l}\text { Trastorno por estrés } \\
\text { postraumático }\end{array}$ & 1 & 3.0 & 1 & 2.6 & $\S$ & 0.71 \\
\hline Abuso de alcohol & 0 & 0 & 2 & 5.2 & $\S$ & 0.28 \\
\hline Anorexia nervosa & 0 & 0 & 1 & 2.6 & $\S$ & 0.53 \\
\hline Bulimia & 3 & 9.0 & 3 & 7.8 & $\S$ & 0.59 \\
\hline $\begin{array}{l}\text { Trastorno de ansiedad } \\
\text { generalizada }\end{array}$ & 15 & 45.4 & 15 & 39.4 & 0.25 & 0.39 \\
\hline
\end{tabular}

se incluyen otras formas de contacto sexual (por ejemplo, los tocamientos); ${ }^{10,11,17}$ algunos estudios, además, 
han considerado el contacto sexual como abuso solamente cuando el perpetrador es al menos cinco años mayor que la víctima, ${ }^{3,11}$ lo cual también puede dar lugar a una reducción en las cifras.

De esta manera, la elevada proporción de mujeres con historia de ASI que encontramos puede deberse a que la definición de AIS que hemos empleado es más incluyente al contemplar una mayor variedad de formas de contacto sexual sin establecer un criterio de edad para el perpetrador del abuso. Cabe señalar, por otro lado, que este hallazgo es congruente con las evidencias que señalan mayor vulnerabilidad para sufrir depresión mayor entre los sujetos con historia de ASI. ${ }^{7}$

Aunque existen pocas dudas sobre la contribución, al menos parcial, del abuso sexual en la aparición subsecuente de episodios depresivos, ${ }^{6,7}$ nuestros resultados sugieren que son pocas las características clínicas que distinguen a las mujeres con depresión e historia de abuso sexual de las que no lo sufrieron. Respecto a la sintomatología depresiva no encontramos diferencias significativas entre los grupos en función de la historia de ASI. Cabe señalar que los estudios disponibles han obtenido resultados mixtos. Gladstone et al. ${ }^{9}$ encontraron en un primer estudio que las mujeres con depresión mayor que habían sufrido ASI mostraban puntuaciones más altas en el IDB, pero no en las apreciaciones clínicas realizadas con la escala para depresión de Hamilton (EHAMD); mientras que en un segundo reporte ${ }^{10}$ del mismo grupo de investigadores con una muestra diferente no encontraron diferencias significativas ni en la EHAMD ni con una escala de 4 puntos (sin depresión a gravemente deprimida).

Zlotnick et al. ${ }^{11}$ tampoco encontraron diferencias significativas en la gravedad de los síntomas depresivos en una muestra compuesta predominantemente por mujeres (85\%). En contraste, Gamble et al., ${ }^{18}$ también con la EHAMD, identificaron que los pacientes con depresión mayor > 50 años con historia de ASI presentaban calificaciones más altas; aunque esta investigación incluyó una muestra equitativa de hombres y mujeres y no se analizó la contribución del sexo.

La inconsistencia en los resultados entre los diferentes estudios no parece obedecer a la forma como se midió el abuso sexual, ya que si bien se emplearon diferentes instrumentos, todos coinciden en definir al abuso sexual como cualquier experiencia no deseada que incluyó contacto físico. De esta forma, este conjunto de hallazgos sugiere que mientras que la experiencia traumática sexual facilita la aparición del trastorno depresivo mayor, su influencia sobre la gravedad de los síntomas depresivos durante los episodios parece ser débil.

En relación con la presencia de otros trastornos psiquiátricos comórbidos encontramos mayor frecuencia de trastorno por ansiedad social entre las mujeres con historia de ASI. Al respecto, la información disponible también es variable. Así, un estudio identificó una frecuencia mayor de trastorno de pánico como trastorno comórbido ${ }^{10}$ mientras que en otro se registró mayor frecuencia de trastorno por estrés postraumático. ${ }^{11}$ Esta variabilidad podría obedecer a que en sí el abuso sexual incrementa el riesgo de psicopatología en general. Aunque también podrían estar influyendo las particularidades de las poblaciones clínicas de cada una de estas investigaciones, ya que en población general la historia del ASI se ha asociado con incremento significativo en el riesgo de presentar justamente estos tres trastornos (trastorno de pánico, trastorno por estrés postraumático y trastorno de ansiedad social) y solamente en las mujeres. ${ }^{5}$ Esto indica que en las mujeres con depresión con historia de ASI es necesario investigar la presencia comórbida de al menos estas tres entidades psiquiátricas.

Dos estudios han evaluado el funcionamiento global en mujeres con depresión sobrevivientes al ASI y, nuevamente, los resultados son mixtos. Ambos emplearon la escala de Evaluación Global del Funcionamiento: uno encontró que las mujeres con el antecedente tenían un funcionamiento significativamente menor, ${ }^{9}$ lo cual no se reprodujo en el otro. ${ }^{11}$

En cuanto al funcionamiento social, Mullen et al. ${ }^{19}$ habían señalado que el abuso sexual en la infancia incrementaba la vulnerabilidad para el desarrollo de dificultades en el funcionamiento social, interpersonal y sexual en la vida adulta de las mujeres. Sin embargo, solamente un estudio ${ }^{11}$ ha evaluado el funcionamiento social en mujeres con depresión sobrevivientes de ASI. Nuestros resultados coinciden con dicha investigación al no encontrar diferencias significativas en el funcionamiento social. De acuerdo con los valores normativos que se han propuesto, las puntuaciones en la EAAS que observamos en uno u otro grupo de mujeres se encuentran por debajo del rango normal (35-52 puntos), correspondiendo a un perfil caracterizado por pocas relaciones extrafamiliares, dificultades de comunicación, poco interés y placer en las actividades que se realizan y dificultades para lograr la independencia; ${ }^{15}$ estas puntuaciones son semejantes 
a las que reportan los sujetos con depresión mayor antes de recibir tratamiento farmacológico.

Es difícil explicar esta ausencia de diferencias en el funcionamiento social, en parte por las múltiples variables que lo determinan. No obstante, es posible que el abuso sexual tenga efecto solamente en componentes particulares del funcionamiento social. En este sentido, se encuentran los hallazgos de Gamble et al., ${ }^{20}$ quienes recientemente informaron que las mujeres con depresión sobrevivientes de ASI que reciben psicoterapia obtienen poca mejoría en el dominio de las relaciones íntimas y con familiares inmediatos, en comparación con otros dominios del funcionamiento social como el trabajo, actividades placenteras con amigos y relaciones extrafamiliares. También es posible que sea la forma como reacciona el sujeto ante la experiencia de abuso la que influya en el funcionamiento social, tal como han indicado Lien y Bouewdy, ${ }^{21}$ quienes identificaron que solamente el grado en que los sujetos se sentían responsables del abuso predecía significativamente el funcionamiento social, en comparación con otras variables como la frecuencia del ASI, la búsqueda de ayuda para afrontar la experiencia y el maltrato en las relaciones adultas. Sin duda, los efectos del ASI sobre el funcionamiento social son un área que requiere mayor investigación.

Además de la mayor comorbilidad con el trastorno por ansiedad social, solamente el riesgo suicida distinguió a las mujeres con depresión sobrevivientes de ASI de las que no lo sufrieron. Este resultado es congruente con los hallazgos de los estudios previos, ${ }^{10,11}$ en los que se ha descrito que las mujeres con depresión e historia de ASI presentan más intentos suicidas y conductas autolesivas. En el estudio longitudinal de la muestra de Zurich, ${ }^{22}$ Angst et al. encontraron que entre las mujeres, el abuso sexual en la infancia fue uno de los predictores más poderosos de la presencia futura de intentos suicidas $(\mathrm{RM}=7.9)$. Asimismo, una revisión sistemática reciente sobre el tema señala que el abuso sexual confiere un riesgo significativo de presentar conducta suicida o conductas autolesivas no suicidas. ${ }^{23} \mathrm{Sin}$ embargo, se trata de un efecto inespecífico y que resulta difícil de aislar de otras variables que comúnmente coexisten con el abuso sexual y que también se asocian con mayor riesgo suicida, como otras formas de maltrato infantil, el ambiente familiar adverso, el trastorno límite de personalidad, entre otros. Nuestros resultados coinciden con este punto de vista al detectar que las mujeres con historia de
ASI también habían sufrido más abuso físico en la infancia, aunque la diferencia no fue significativa.

Identificar que la historia de ASI en las mujeres con depresión mayor se asocia con mayor riesgo suicida no es un hallazgo menor ya que puede tener implicaciones terapéuticas. Por tanto, la investigación de historia de abuso sexual en la infancia debe realizarse de manera sistemática en las mujeres con depresión mayor.

Este estudio muestra algunas limitaciones que deben considerarse al interpretar los resultados. Las características de la muestra como el número de participantes, el sexo, su selección por conveniencia y de una institución de atención primaria limitan la generalización de los resultados a otras poblaciones. Otra limitación es que se trata de un estudio transversal, por lo que los resultados solamente corresponden al momento de la evaluación y no pueden realizarse inferencias causales, por ejemplo, entre la historia de abuso sexual en la infancia y el riesgo suicida. Debe también tenerse en cuenta que la investigación sobre información del pasado de una persona está sometida al sesgo del recuerdo y que, cognitivamente hablando, los individuos con depresión tienden a evocar más los recuerdos negativos. No obstante, también se ha observado que el recuerdo de experiencias emocionales es más confiable durante periodos en que el estado de ánimo es congruente con el contenido emocional de dichos recuerdos. ${ }^{24}$ En este mismo sentido debemos reconocer que obtener información clínica mediante autorreporte puede conducir al subregistro, en particular respecto al abuso sexual en la infancia por tratarse de un tema embarazoso para el sujeto, ${ }^{25}$ aunque esto también ocurre en proporciones similares con otros formatos como la entrevista, ${ }^{26}$ con la que existe concordancia. ${ }^{27}$

\section{Bibliografía}

1. Finkelhor D. The international epidemiology of child sexual abuse. Child Abuse Negl. 1994;18:409-417.

2. Rivera-Rivera L, Allen B, Chávez-Ayala R, Avila-Burgos L. Abuso físico y sexual durante la niñez y revictimización de las mujeres mexicanas durante la edad adulta. Salud Publica Mex. 2006;48(Supl. 2):S268-S278.

3. Villatoro-Velázquez J, Quiroz-Del-Valle N, Gutiérrez-López ML, Díaz-Santos M, Amador-Buenabad N. ¿Cómo educamos a nuestros/as hijos/as? Encuesta de Maltrato Infantil y Factores Asociados 2006. México: Instituto Nacional de las Mujeres/Instituto Nacional de Psiquiatría Ramón de la Fuente Muñiz; 2006.

4. Chen LP, Murad MH, Paras ML, Colbenson KM, Sattler AL, Goranson EN, et al. Sexual abuse and lifetime diagnosis of psychiatric disorders: systematic review and meta-analysis. Mayo Clin Proc. 2010;85:618-629.

5. Molnar BE, Buka SL, Kessler RC. Child sexual abuse and subsequent psychopathology: results from the National Comorbidity Survey. Am J Public Health. 2001;91:753-760.

6. Kendler KS, Aggen SH. Clarifying the causal relationship in women between childhood sexual abuse and lifetime major depression. Psychological Med. 2014;44:1213-1221. 
7. Maniglio R. Child sexual abuse in the etiology of depression: a systematic review of reviews. Depression Anxiety. 2010;27:631-642.

8. Boudewyn AC, Liem JH. Childhood sexual abuse as a precursor to depression and self-destructive behavior in adulthood. J Traumatic Stress. 1995;8:445-459.

9. Gladstone G, Parker G, Wilhelm K, Mitchell PB, Austin MP. Characteristics of depressed patients who report childhood sexual abuse. Am J Psychiatry. 1999;156:431-437.

10. Gladstone GL, Parker GB, Mitchell PB, Malhi GS, Wilhelm K, Austin MP. Implications of childhood trauma for depressed women: an analysis of pathways from childhood sexual abuse to deliberate self-harm and revictimization. Am J Psychiatry. 2004;161:1417-1425.

11. Zlotnick C, Mattia J, Zimmerman M. Clinical features of survivors of sexual abuse with major depression. Child Abuse Negl. 2001;25:357-367.

12. Sheehan DV, Lecrubier $Y$, Sheehan KH, Amorin $P$, Janavs J, Weiller $E$ et al. The MINI-International Neuropsychiatric Interview (MINI): the development and validation of a structured diagnostic interview for DSM-IV and ICD-10. J Clin Psychiatry. 1998;59(Suppl 20):22-23.

13. Beck AT, Steer RA. Beck Depression Inventory Manual. EE. UU.: Psychological Corporation; 1993.

14. Ibarra-Alcantar MC, Ortiz-Guzmán JA, Alvarado-Cruz FJ, Graciano-Morales $\mathrm{H}$, Jiménez-Genchi $\mathrm{A}$. Correlatos del maltrato físico en la infancia en mujeres adultas con trastorno distímico o depresión mayor. Salud Mental. 2010;33:317-324.

15. Bosc M, Dubini A, Polin V. Development and validation of a social functioning scale, the Social Adaptation Self-evaluation Scale. European Neuropsychopharmacol. 1997;7 (Suppl 1):S57-S70.

16. Bobes J, González MP, Bascarán MT, Corominas A, Adan A, Sánchez J, et al. Validación de la versión española de la Escala de Adaptación Social en pacientes depresivos. Actas Esp Psiquiatr. 1999;27:71-80.

17. Ramos-Lira L, Saltijeral-Méndez MT, Romero-Mendoza M, Caballero-Gutiérrez MA, Martínez-Vélez NA. Violencia sexual y problemas asociados en una muestra de usuarias de un centro de salud. Salud Publica Mex. 2001;43:182-191.

18. Gamble SA, Talbot NL, Duberstein PR, Conner KR, Franus N, Beckman AM, et al. Childhood sexual abuse and depressive symptom severity: the role of neuroticism. J Nerv Ment Dis. 2006;194:382-385.

19. Mullen PE, Martin JL, Anderson JC, Romans SE, Herbison GP. The effect of child sexual abuse on social, interpersonal and sexual function in adult life. Br J Psychiatry. 1994;165:35-47.

20. Gamble SA, Smith PN, Poleshuck EL, He H, Talbot NL. Domain-specific social functioning improvements during treatment of depressed women with histories of childhood sexual abuse. J Affect Disord. 2011;130: 478-482.

21. Liem JH, Boudewyin AC. Contextualizing the effects of childhood sexual abuse on adult self and social functioning: an attachment theory perspective. Child Abuse Negl. 1999;23:1141-1157.

22. Angst J, Hengartner MP, Rogers J, Schnyder U, Steinhausen HC, Adjacic-Gross V, et al. Suicidality in the prospective Zurich study: prevalence, risk factors and gender. Eur Arch Psychiatry Clin Neurosic. 2014;264: 557-565.

23. Maniglio R. The role of child sexual abuse in the etiology of suicide and non-suicidal self-injury. Acta Psychiatr Scand. 2011;124:30-41.

24. Ellis HC, Moore BA. Mood and memory. En: Dalgleish T, Power MJ, eds Handbook of cognition and emotion. EE. UU.: John Wiley \& Sons; 1999.

25. Henry B, Moffitt TE, Caspi A, Langley J, Silva PA. On the "remembrance of things past": a longitudinal evaluation of the retrospective method. Psychol Assess. 1994;6:92-101.

26. Williams LM. Recall of childhood trauma: a prospective study of women's memories of child sexual abuse. J Consult Clin Psychol. 1994;62:1167-1176.

27. Fink LA, Bernstein D, Handelsman L, Foote J, Lovejoy M. Initial reliability and validity of the childhood trauma interview: a new multidimensional measure of childhood interpersonal trauma. Am J Psychiatry. 1995; 152:1329-1335.

\section{Anexo \\ Índice de Abuso Sexual Infantil}

¿Entre los 3 y 17 años, alguna persona la indujo a realizar alguna de las siguientes acciones?

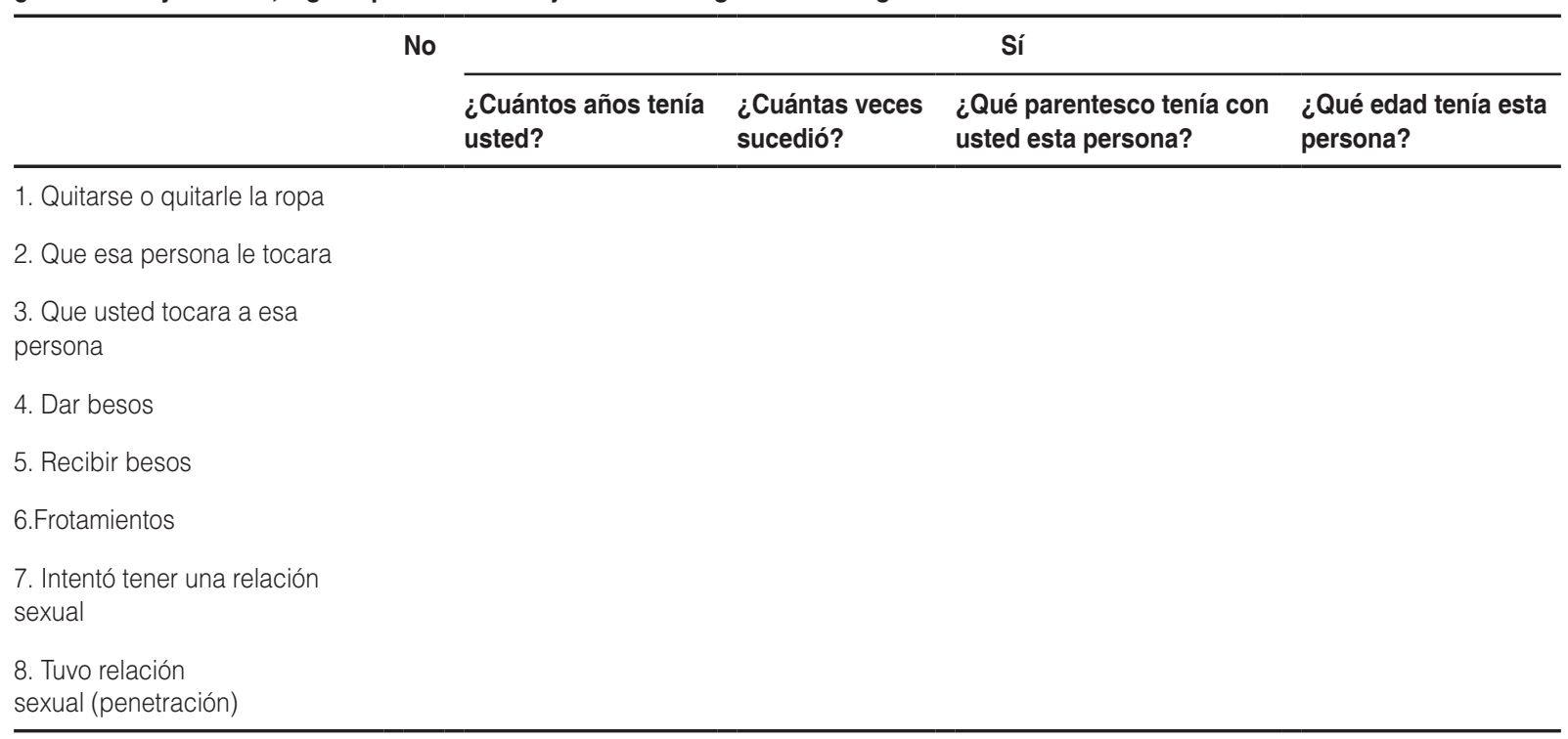

